\title{
The dynamics of second- and third-male fertilization precedence in Tribolium castaneum
}

\author{
Arnaud $\mathrm{L}^{[1]}$, Gage $\mathrm{M}^{[2]}$, Haubruge $\mathrm{E}^{[1]}$ \\ [1] Unit of General and Applied Zoology, Gembloux Agricultural University, B-5030 Gembloux, \\ Belgium; \\ [2] Population \& Evolutionary Biology Research Group, School of Biological Sciences, Nicholson \\ Building, University of Liverpool, Liverpool L69 3BX, UK
}

\section{Key words}

sperm competition, spermatozoa, spermatheca, mating

\begin{abstract}
Sperm precedence is a useful descriptor of the outcome and possible processes of sperm competition between (usually) two males. Although sperm precedence values are known for a variety of taxa, a characteristic feature has been the profound, and largely unexplained, variance in precedence. In this study we partition some of the precedence variance in Tribolium castaneum (Herbst) (Coleoptera, Tenebrionidae) by examining how: (1) sperm precedence changes with time over a one-month oviposition period, (2) precedence is influenced by the relative timing of the two competitive mating periods, and (3) sperm precedence is affected when three, rather than the normal two, males are placed in sperm competition. In general, last male sperm precedence is high $(\approx 90 \%)$ for the first few days after mating, but this changes significantly with time as previous males' sperm become used in fertilization. We find that the duration between two competitive mating bouts has a significant effect on precedence (even when relatively brief): longer intervals between mating bouts result in more complete and persistent last-male sperm precedence. The dynamics of last-male sperm precedence when there are three males in the competition are not significantly different to precedence results when only two males are in competition. Our results are consistent with the hypothesis that, soon after inseminations, male ejaculates 'stratify' in the narrow and elongate storage tubules of the spermatheca. With time we see increased variance in individual male precedence as sperm from previous matings achieve more fertilizations. This change over time may result from depletion (either via fertilizations or passive/active sperm loss) of the last males' sperm and/or increased mixing of different males' sperm within the spermatheca.
\end{abstract}

\section{Introduction}

The competition between different males' sperm to fertilize a female's ova has been a potent force in the evolution of male reproductive morphology, physiology and behaviour (Birkhead \& Møller, 1998). Despite widespread recognition of the influence of sperm competition, the actual processes and mechanisms by which sperm compete are less well understood. In this study we investigate the dynamics of sperm competition in the red flour beetle Tribolium castaneum: (1) when there is variation in the interval between the competing mating periods; (2) when both two and three males' sperm are in competition; and (3) we track fertilization patterns for one month after the onset of sperm competition. In the natural environment of stored products, $T$. castaneum mating patterns are dependent on local population structure and both sexes have high propensities to multiple mating (Sokoloff, 1974; Haubruge et al., 1999). Given the potential variance in each population's mating pattern we have chosen three intermating intervals that represent the variance from extreme polygamy in high density populations to rare mate encounter in isolated, low density populations. In the majority of insect species it is the second male to mate in a sequence that fertilizes most of a female's eggs (Simmons \& Siva-Jothy, 1998). This is termed second-male sperm precedence [often cited as P2: the proportion of offspring sired by the second male to mate (Boorman \& Parker, 1976)] 
and represents the fertilization outcome of sperm competition between the two males. Although mean P2 is a useful value for describing the outcome of sperm competitions, it is important to note that P2 is a variant that is influenced by a number of potentially-interacting factors. Simmons \& Siva-Jothy (1998) show that P2 is an extremely variable descriptor; in their recent review of more than 100 insect species they demonstrate that within-species variance in $\mathrm{P} 2$ is as extensive as the across-species variance. Indeed, it was a study of T. castaneum by Lewis \& Austad (1990) that was one of the first to recognise and explore the importance of variance in P2. It is therefore important to conduct experimental studies which partition variance in P2 in an attempt to interpret causal mechanisms. In this study we examine male fertilization precedence in $T$. castaneum over a longer post-mating time frame and we compare precedence dynamics when the time-period between competitive mating bouts is varied. In T. castaneum both sexes mate repeatedly (Sokoloff, 1974; Haubruge et al., 1999) but some do not result in successful sperm transfer (unpublished data show that of 140 apparently normal matings only 81 transferred sperm). We therefore allow single males a longer duration of mating access to females and promote successful sperm transfer (see Results). Although this longer mating access potentially introduces variance from uncontrolled mating frequencies, longer-term mating access is a more likely phenomenon in the natural environment of grain stores where males can mate repeatedly with the same, or different, females (Haubruge et al., 1999). Furthermore, we overcome the potential variance between males in mating frequency by using randomly-selected control males with identical mating history and stock derivation. Accordingly, our results explore the dynamics of fertilization precedence after mating bouts, rather than single ejaculates.

In most insects females store sperm in a specialised organ (the spermatheca) often for prolonged periods (e.g., years in some hymenopterans, Tschinkel, 1987). It is therefore important to measure the temporal variance in sperm precedence that occurs with an increasing duration of sperm storage (e.g., Ueno \& Ito, 1992; Yamagishi et al., 1992; Siva-Jothy \& Tsubaki, 1989; 1994; Siva-Jothy et al., 1996). The longer-term changes can provide informative insights into the mechanisms of sperm competition. In $T$. c a s $t$ a -neum females can lay fertile eggs from a single mating for up to 140 days (Bloch Qazi et al., 1996) and therefore it is important to track fertilization precedence over longer oviposition periods. Schlager's (1960) pioneering study on sperm precedence in T. castaneum was one of the first to examine sperm precedence from competing males' sperm over a relatively longer time-frame. Schlager showed that the last male to mate preceded previous matings. Our study is a continuation and development of Schlager's (1960) early investigation and we track how sperm precedence in $T$. castaneum varies over a one month period after the onset of sperm competition.

In addition to post-mating temporal variance, fertilization precedence may also be influenced by variation in the relative timing of competing matings. Post-insemination processes involving sperm migration, mixing, and storage may influence the subsequent dynamics of fertilization precedence. For example in the silk moth Bombyx mori, sperm precedence by the second male is very high (mean $\mathrm{P} 2=$ $0.95)$ when the inter-mating interval is $2 \mathrm{~h}$, but this drops to very low second male precedence $(\mathrm{P} 2=$ 0.06) when the second mating follows immediately (Suzuki et al., 1996). This change in P2 is probably caused by the timing of sperm transfer from the spermath-ophore to the spermatheca and from the spermatheca to the vestibulum (Suzuki et al., 1996). Again, the variation in fertilization precedence under different pre-competition inter-mating intervals can provide important insights into mechanisms of sperm transfer and competition (Boorman \& Parker, 1976; Gwynne \& Snedden, 1995). We therefore examine long-term dynamics in sperm precedence in $T$. castaneum when intervals between the two competitive mating periods are zero, one day, and 14 days.

Most sperm competition studies investigate fertilization precedence between only two males that have mated with the female sequentially (review in Simmons \& Siva-Jothy, 1998). In nature this level of polyandry may not be the norm but few studies have investigated the outcome of sperm competition between more than two males. In the pseudoscor-pion Cordylochernes scorpioides, the two-male sperm precedence pattern breaks down when a third male is introduced to the competition (Zeh \& Zeh, 1994). However, in the bruchid Callosobruchus maculatus last males achieve similar last-male precedence patterns whether two or three males enter the competition (Eady \& Tubman, 1996). In $T$. castaneum females behave polyandrously (Sokoloff, 1974) and it is therefore appropriate to examine P3 and beyond. In T. c a s $t$ a-neum, Lewis \& Jutkiewicz (1998) show that third-male precedence is similar in magnitude to second-male values: both are approximately 0.7 in the first week after mating and this declines over two subsequent weeks as the first males' sperm become prevalent. We run similar competitions, but we perform reciprocal crosses so that we can track the dynamics in first-, second-, and third-male sperm precedence together and measure in detail over a one month period postmating. Last male sperm precedence may arise through a variety of possible mechanisms including sperm removal (Waage, 1979), sperm displacement (Simmons \& Parker, 1992), stratification of sperm in storage (Schlager, 1960), optimal ejaculate positioning and passive or active sperm loss from the 
female tract (Etman \& Hooper, 1979; Otronen, 1997), sperm ageing (Yamagishi et al., 1992), numerical (Simmons, 1987; Cook et al., 1997) or physiological superiority of sperm, genetic compatibility (Wilson et al., 1997), and cryptic female choice (Ward, 1993; Eberhard, 1996). By varying the interval between mating bouts, examining three males in competition, and tracking the dynamics of sperm precedence over a longer time frame, we are able to explore in more detail how mechanisms of sperm precedence arise and develop in T. castaneum.

Tribolium castaneum is a model species for investigations of sperm competition. Females mate polyandrously (Sokoloff, 1974) and store sperm in a tubular spermatheca (Sinha, 1953). Double-male precedence studies show that $T$. castaneum males that are second to mate achieve fertilization precedence (P2 averages 0.6, Schlager, 1960; Wool \& Bergerson, 1979; Lewis \& Austad, 1990, 1994) but that there can be considerable variation between males in the degree of precedence (Lewis \& Austad, 1990). Furthermore, Bloch-Qazi et al. (1996) provide important details of sperm transfer and storage dynamics in this flour beetle that can be related to sperm precedence results. Within this informative framework, therefore, we investigate longer term sperm precedence dynamics in $T$. castaneum using both two-male and three-male sperm precedence experiments, and with variable periods between the competitive mating periods.

\section{Materials and methods}

\section{Tribolium strains}

We used two flour beetle strains that were resistant and susceptible to malathion. The resistant strain (PRm) was originally derived from grain stores in the Philippines. The susceptible strain (Asm) was derived from storage facilities in the Ivory Coast. Both strains were cultured in a dark incubator, at 30 $\pm 3 \circ \mathrm{C}$ and $60 \pm 5 \%$ r.h. with a mixture of whole wheat flour and brewer's yeast (10/1-wt/wt) as rearing medium. Paternity was assigned using malathion-specific resistance which is an autosomal, semidominant and monofactorial genetic marker with no evidence for segregation (Beeman, 1983). There is a significant difference between the PRm and Asm strains in mean sperm precedence (mean precedence for $P R m=0.85( \pm 0.008 \mathrm{SE})$ and $A s m=0.72( \pm 0.099 \mathrm{SE})$; General Linear Model, F1, $661=$ $42.48, \mathrm{P}<0.0001$ ). However, genotype mating-order was randomised throughout and completely reciprocated which controls fully for any between-strain differences in competitive ability.

\section{Rearing and experimental protocols}

Randomly-selected pupae from stock populations were sexed and maintained as the stock culture but were individually isolated. Adults were one month old at the start of the experiments. Males were marked for identification on the elytra. Matings were conducted by placing a pair of beetles in a vial with $0.5 \mathrm{~g}$ of rearing medium for a $48 \mathrm{~h}$ mating period. During this period, pairs were free to mate. This period allows for successful sperm transfer which is not guaranteed by observing the act of copula; we find that using this mating bout period increases successful sperm transfer from a single male from $58 \%$ to $95 \%(\mathrm{~N}=140$ and 180 matings, respectively). After a determined interval following the mating period (depending upon the experiment), females were placed in a new vial with another male for the same mating period. No individual was used more than once in any experiment. The same method was repeated for three-male experiments. The $0.5 \mathrm{~g}$ of medium was then added to a $55 \mathrm{~mm}$ Petri dish with a further $4.5 \mathrm{~g}$ of rearing medium. Females were then maintained individually in petri dishes with $5 \mathrm{~g}$ of rearing medium and transferred to a fresh dish with new rearing medium every three days for 30 days. These transfers reduced potential cannibalism among different aged larvae (Lewis \& Austad, 1990) and enabled us to track detailed variance in paternity with time. Only susceptible females were used since mating with resistant females gives only resistant progeny whatever the male phenotype.

Table 1. Reciprocal crosses to discriminate paternity of first-, second-, and third-male in triple-mating experiments using resistant $(R)$ and susceptible $(S)$ genotypes

\begin{tabular}{lllll}
\hline \multirow{2}{*}{$\begin{array}{l}\text { Reciprocal } \\
\text { crosses }\end{array}$} & \multicolumn{3}{l}{ Male phenotype } \\
\cline { 3 - 5 } & & Male1 & Male2 & Male 3 \\
\hline P1 & $1)$ & $R$ & $S$ & $S$ \\
& $2)$ & $S$ & $R$ & $R$
\end{tabular}




$\begin{array}{lllll}\mathrm{P} 2 & 1) & S & R & S \\ & 2) & R & S & R \\ \mathrm{P} 3 & 1) & S & S & R \\ & 2) & R & R & S\end{array}$

In all crosses, mating order of the two genotypes was randomised and reciprocated (Table 1) to eliminate any strain-associated fertilization bias.

After 45 days, adult progeny of every single female was sifted from each $5 \mathrm{~g}$ of rearing medium and paternity was assigned using a discriminating-dose, malathion contact bioassay: all susceptibles die while resistants survive (Haubruge et al., 1997). Malathion-specific resistance is semidominant (Beeman, 1983) and this test therefore enables the identification of the male parent phenotype and hence the degree of fertilization precedence in two-male and three-male experiments. Differential larval survival in the standard and non-competitive culture conditions of this study is not likely to confound our precedence results: we used reciprocal crosses to randomise male mating order and, in the absence of insecticide challenge, there is no evidence that insecticide resistance generates significant differences in larval survival in other insects (e.g., McKenzie \& O'Farrell, 1993). In all precedence experiments, 30 females were used in each treatment (i.e., 90 females were used in the two-male inter-mating interval experiments). Occasionally, females died or did not complete the entire one month oviposition period successfully and these females were omitted from analyses.

\section{Two-male sperm precedence experiments}

Resistant $(P R m)$ and susceptible $(A s m)$ male pairs were sequentially given mating access to a susceptible (Asm) virgin female either with (i) no intermating bout interval (females were transferred immediately from one male to the next), (ii) one day, or (iii) 14 days mating bout interval. Reciprocal crosses of the two males were conducted to control for any potential strain differences. Thirty females were mated in each of the three inter-mating interval trials (15 male PRm X male Asm matings; 15 male Asm X male PRm matings).

\section{Three-male sperm precedence experiments}

A male trio, comprising two PRm and one Asm males or one PRm and two Asm males, were sequentially given mating access to an $A s m$ virgin female with no inter-mating bout intervals (see Table 1). By using all combinations of the two male genotypes when three males were mating in competition, we were able to identify the paternity of $\mathrm{P} 1, \mathrm{P} 2$, and $\mathrm{P} 3$ using reciprocal crosses. In other words, for P1 we use the following two mating sequences: PRm-Asm-Asm and Asm-PRm-PRm, for P2 we use PRmAsm-PRm and Asm-PRm-Asm, and for P3 we mate Asm-Asm-PRm and PRm-Asm-Asm in sequence. These reciprocal crosses using controlled genotype sequences (detailed in Table 1) enable us to make independent measures of paternity that are not confounded by any differences in competitiveness of the PRm or Asm genotype.

In two-male experiments, the success of each first mating was established by checking the mating vials for the presence of progeny following the first mating period ( 85 of 90 successfully oviposited). Since unsuccessful second matings could not be distinguished from complete first-male sperm precedence, second-male sperm precedence values of zero during the entire experimental period (5 of 90 trials) were not considered in statistical analyses. Since progeny were monitored directly over a one month oviposition period after mating access, it is unlikely that successful sperm transfer would result in zero fertilizations for a male. In three-male experiments, first and third male mating successes were tested as described above and zero fertilization successes over the entire experimental period were omitted from further testing. However, it was not possible to establish unambiguous second male mating success in the three-male experiments since only two genotypes were available; it is unlikely that this influenced our three-male precedence experiment results since pooled total precedence values for the first-males, second-males and third-males summed to a mean of 0.988 ( $\pm 0.051,95 \%$ confidence limits; no significant departure from $100 \%)$.

For comparisons of two-male and three-male precedence results over time under different treatments we use repeated measures GLM. Although we present untransformed data for clarity (mean \pm standard error), we normalised all proportional precedence data before statistical analysis using angular transformation (Dagnelie, 1998). 


\section{Results}

\section{Two-male precedence}

The progress of second-male precedence for each of the three inter-mating intervals is presented in Figure 1. Each data point represents mean second-male precedence $( \pm \mathrm{SE})$ for all females over each three day oviposition period. In all three inter-mating interval experiments, second-male sperm precedence is maximum in the first three days following mating and then declines regularly from day 3 to 30 .

There were significant differences between the three inter-mating interval treatments in overall precedence (analysing across each entire 30 day oviposition period): GLM, F2,661 $=41.70, \mathrm{P}<$ 0.0001 . Multiple comparisons (Tukey's test): (i) 0 h versus one day: $t_{631}=40.16, \mathrm{P}=0.0001$; (ii) $0 \mathrm{~h}$ versus 14 days: $t_{631}=9.13, \mathrm{P}<0.0001$; (iii) one day versus 14 days: $t_{631}=4.45, \mathrm{P}<0.0001$ ). Mean $( \pm \mathrm{SE})$ second-male precedence values for each of the three inter-mating intervals were: 0 day interval $\mathrm{P} 2=0.69( \pm 0.02, n=235)$; one day interval P2 $=0.82( \pm 0.018, n=224) ; 14$ day interval $\mathrm{P} 2=0.95( \pm$ $0.01, n=217)$.

We present the development of second-male precedence variance for all three inter-mating interval treatments in Figure 2. Variance declines significantly over the first three days when last-male precedence is at its highest (comparing day 0 to day $3 \mathrm{~F}_{77,75}=1.91, \mathrm{P}=0.0187$ ) and then increases significantly from day 3 to day $6\left(\mathrm{~F}_{73,75}=1.84, \mathrm{P}=0.0302\right)$ and thereafter, although it was not different from day 6 to day 30 (maximum $F$ for all comparisons: $F_{69,73}=1.57, P=0.1720$ ), precedence variance shows a steady increase up to day 30 .

\section{Comparing two-male and three-male precedence values}

Three-male sperm precedence values were obtained by mating females sequentially with no intermating interval. It is therefore appropriate to compare three-male values with the two-male values which were obtained using a similar pre-final mating time period to limit the possible effects of sperm depletion which could arise from variance in the temporal opportunity to oviposit. We therefore compare (last male) three-male values with the two-male values produced when females were allocated a one-day inter-mating interval. Thus three-male females have a maximum of $144 \mathrm{~h}(3 \times 48 \mathrm{~h})$ from first to final insemination; two-male values with the one-day mating interval have a maximum of $120 \mathrm{~h}$ from first to last insemination. Relating oviposition results for these two female groups suggests that this is the most appropriate comparison: we find that similar relative numbers of offspring are produced ('oviposited') in the period before the final competitive mating: pre-two-male (one-day interval) mating $=9.29 \%$, pre-three-male mating $=9.33 \%$. In contrast only $3.43 \%$ of the progeny were produced in the pre-two-male mating when there was a zero inter-mating interval. Furthermore the proportions of offspring sired by the first male(s) are similar for two-male one-day interval and three-male matings (32.45\% and $32.73 \%$, respectively) whereas the first males in the 0 -day interval two-male treatments sired only $9.40 \%$ of the total progeny. Accordingly, when we compare (one-day interval) two-male precedence results with three-male mating bouts we find no significant differences between the range of precedence values (GLM, F1435 $=0.41, \mathrm{P}=0.524$, compare Figure 1 one-day interval plot with Figure 3).

\section{Three-male precedence}

Figure 3 illustrates the changes in the first-, second-, and third-males' precedence over time. Over the first three days second-male precedence is significantly greater than first-male precedence (GLM, day $0: \mathrm{F} 151=14.80, \mathrm{P}<0.0001$; day $3: \mathrm{F} 151=5.74, \mathrm{P}=0.020)$. After the first three days however, there are no significant differences between the first-male and second-male precedence values except for day $30(\mathrm{GLM}, \mathrm{F} 130=4.81, \mathrm{P}=0.030)$. Mean precedence values for all three males averaged across all oviposition periods are respectively $0.112( \pm 0.016), 0.087( \pm 0.012)$, and $0.790( \pm 0.017)$ for first, second and third males. 


\section{Discussion}

Sperm precedence is a variable descriptor of the outcome of sperm competitions (Lewis \& Austad, 1990; Cook et al., 1997; Simmons \& Siva-Jothy, 1998). Our study partitions some of this variance into precedence changes that may occur with time both before and after the competitive matings. In our two-male precedence experiments we demonstrate that the time interval between the two competitive matings has a significant effect upon subsequent precedence, even when relatively brief compared with the time of either mating bout. We also show that the precedence of the second male's sperm decline with time as the first male's sperm become successful in fertilization.

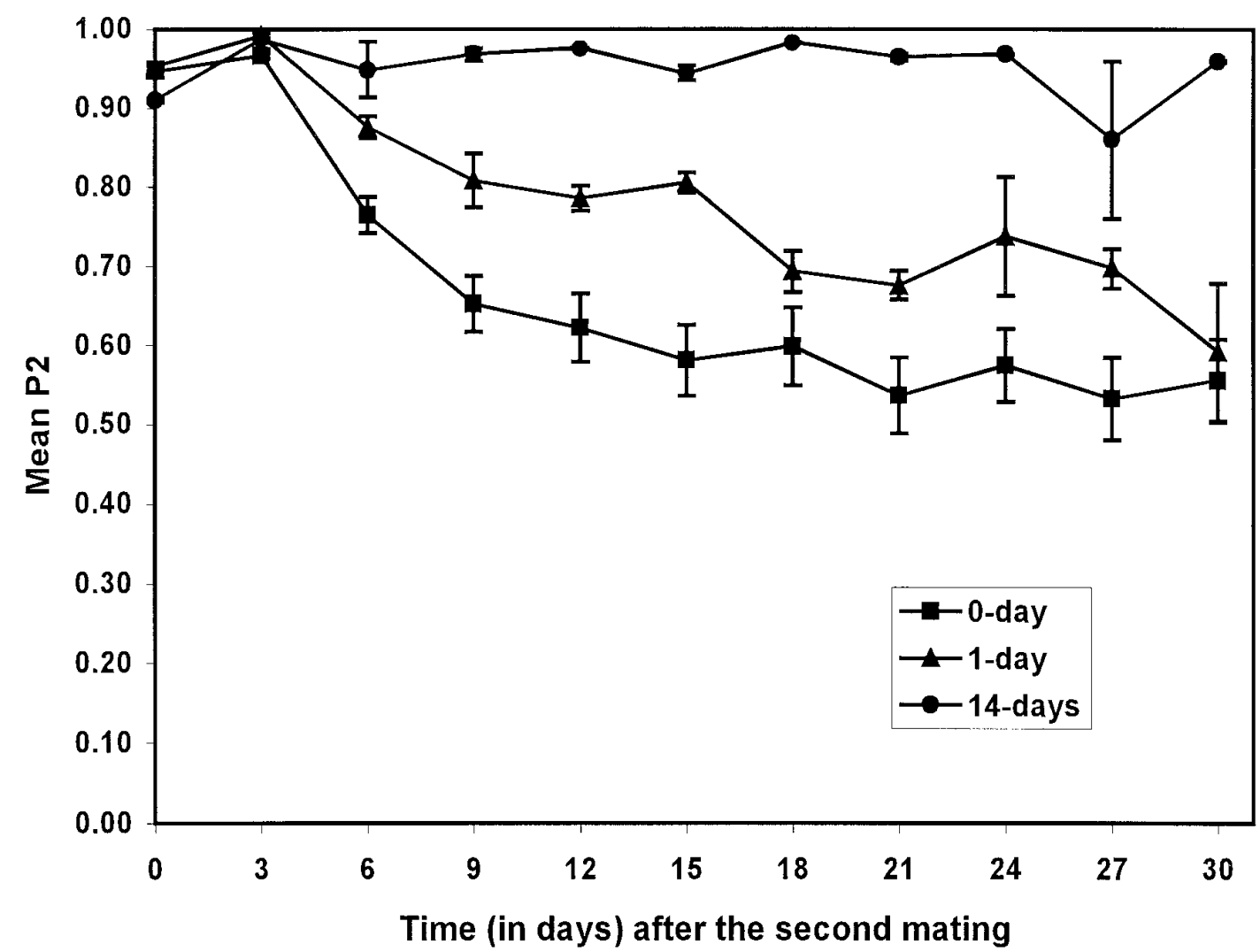

Figure 1. Dynamics of sperm precedence over 30 days of oviposition after the second mating. The three lines represent sperm precedence from inter-mating intervals of 0, 1, and 14 days, respectively. Each data point is the mean ( \pm SE) from an average of 20.55 ( \pm 1.82$), 21.36( \pm 1.03)$ and 20.00 ( \pm 1.18) females (mean $\pm S E$ ) for 0 , 1, and 14 days inter-mating intervals respectively.

We develop sperm competition studies and detail the dynamics of precedence when sperm from a third male enter the competition: final male precedence is not significantly different when two or three males are in sperm competition when measured using similar experimental protocols.

In the double-mating experiments, our results show that second-male precedence is highest when there is a greater interval between the two matings (Figure 1 and means in Results). This is significant even when the inter-mating interval is only $24 \mathrm{~h}$ (which is relatively brief compared with the $48 \mathrm{~h}$ mating bouts). It seems likely that the persistently high levels of second-male precedence in the 14 day intermating interval treatments (Figure 1 shows no evidence for a decline in precedence over 30 days) is the result of previous males' sperm depletion or utilization; females may have used much of the previous males' sperm in storage while ovipositing over the 14days leading up to the second mating. Interestingly, we find a significant difference in the level and development of sperm precedence when the inter-mating interval differs by only one day despite each male having $48 \mathrm{~h}$ mating access to the female (Figure 1, comparing 0-day and one-day inter-mating interval treatments). It appears unlikely that this difference is solely the result of increased sperm depletion over the 1 day inter-mating interval when females were able to oviposit: fertile eggs can continue to be laid for 140 days after a single mating (Bloch-Qazi et al., 1996) therefore significant sperm depletion is unlikely to occur over this brief period which represents less than $1 \%$ of the total duration of sperm storage. Sperm removal is also 
unlikely to be the mechanism of sperm precedence involved here (despite evidence for removal in $T$. castaneum, Haubruge et al., 1999) since migration of sperm into storage occurs over $2 \mathrm{~h}$ postcopulation and we might expect removal to be more effective when sperm are vulnerable to removal at the site of insemination in the copulatory bursa. In T. castaneum the aedeagus cannot access the spermatheca (Haubruge et al., 1999).

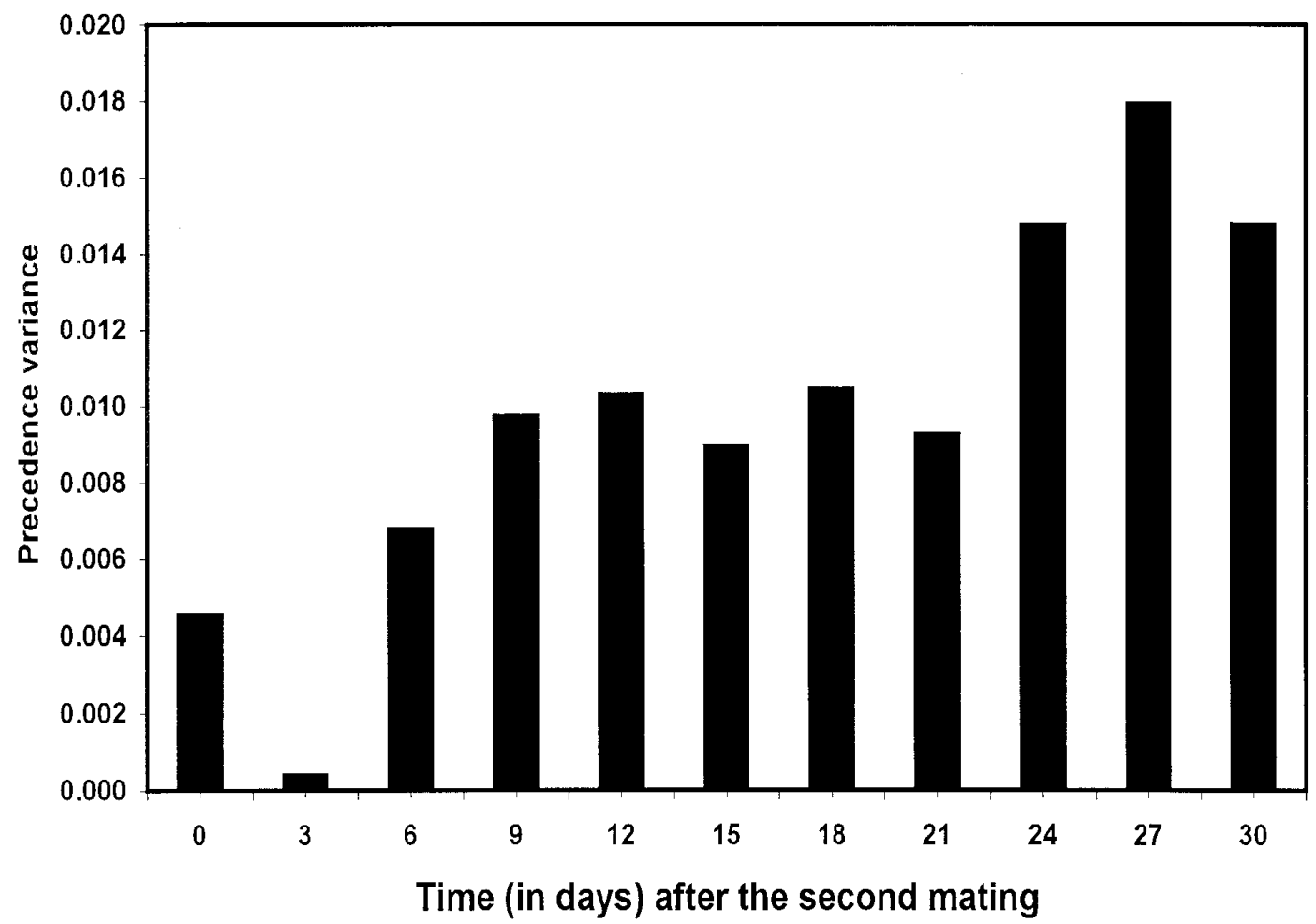

Figure 2. Variance in second-male precedence increases with time after the second mating as both males' sperm are used in fertilizations (see Results for statistical comparisons). The mean variance is derived from all three inter-mating interval treatments.

We find that last male precedence is greater when there is an increased interval between mating periods; if sperm removal occurs the reverse would be expected. Sperm displacement is another possible mechanism by which sperm precedence can arise (Simmons \& Parker, 1992; Eady, 1994). However, since we measure the long-term changes in precedence we can demonstrate that, with time, sperm from previous males become more prevalent in fertilization and therefore were not previously displaced out of storage. It therefore seems unlikely that displacement of sperm from the female reproductive tract explains our results. Precedence via displacement would be manifested by a lastmale fertilization precedence that does not decline over time.

Our results are consistent with Schlager's (1960) pioneering hypothesis that in T. castaneum sperm precedence occurs via a mechanism of stratification of sperm in the narrow and elongate tubules of the sper-matheca (Sinha, 1953). When different males' inseminations are separated by time, there is reduced ejaculate mixing in the bursa since sperm have migrated from the bursa into spermathecal storage (Bloch-Qaziet al., 1996). The spermatheca is a cul-de-sac and has a single entrance duct through which sperm also exit (Sinha, 1953; Schlager, 1960). Subsequent ejaculates enter storage but may be positioned behind any previous males' sperm; there is thus a last-in first-out mechanism of storage and fertilization. However, when different males' inseminations are not separated by time there may be ejaculate mixing in the bursa prior to spermathecal migration and hence a more heterogenous mixing of sperm through the spermath-ecal tubules. Such a mechanism would be manifested as more pronounced second-male sperm precedence when insemination periods are separated by time, and lower precedence when different males' ejaculates are not separated by time and mix in the bursa before storage. With time into the oviposition period, sperm may mix within the spermatheca and/or the last males' sperm become depleted via fertilizations. Last-male precedence should therefore decrease with time as the first males' sperm become used in fertilizations. We chart a clear increase in the variance of precedence as time continues since the competitive matings (Figure 2). This increased 
variance in precedence may reflect a decrease in ejaculate competition (and limited sperm mixing) and an increase in sperm competition as individual sperm mix and compete within the spermatheca.

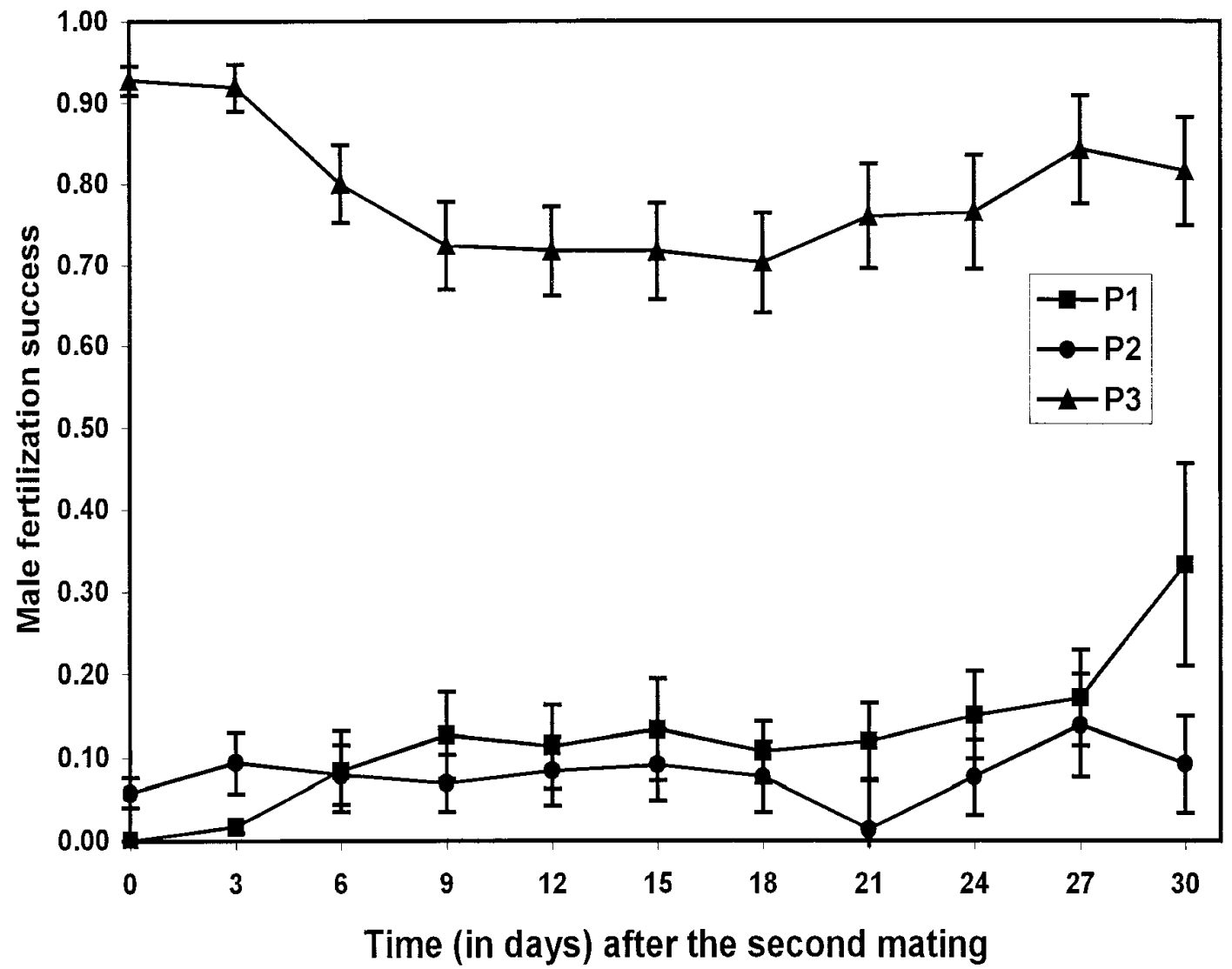

Figure 3. Dynamics of first-, second-, and third-male fertilization precedence with time (following the third mating). Last male sperm precedence is not significantly different when three males are in competition as when two males are in competition (compare with Figure 1). Third-male precedence declines slowly while first-and second-male precedence increase towards the end of the 30 day oviposition period. We attempted to sample 30 females at each data point but some did not oviposit or died prematurely. Mean sample size ( \pm SE) therefore is $18.82( \pm 2.20), 25.00( \pm 0.93)$, and $22.64( \pm$ 1.07), respectively for first, second, and third males.

Our two-male precedence results comparing 0-day and one-day inter-mating intervals (Figure 1) are consistent with the above hypothesised mechanism of sperm transfer, storage, competition, and fertilization. Even though each male was allowed unlimited mating access for $48 \mathrm{~h}$, there is a clear difference in two-male fertilization precedence between situations when the second male is immediately given mating access to the female compared with when there is an interval of $24 \mathrm{~h}$ (Figure 1). This clear result suggests that there was not significant between-male variance in the timing or frequency of copulations over each $48 \mathrm{~h}$ mating bout.

In the related Tenebrio molitor P2 is initially high (ca. 0.9) and then decreases with time since the onset of competition (Siva-Jothy et al., 1996) in a similar manner to T. castaneum. However, Siva-Jothy et al. (1996) found that different inter-mating intervals ( $>10 \mathrm{~min}$ or $24 \mathrm{~h}$ ) have no influence on subsequent P2 in T. molitor. Spermatophore evacuation and sperm transfer to storage appears similar for both species (average sperm transfer to the spermatheca peaks at around $60 \mathrm{~min}$; Bloch-Qazi et al., 1996 and Gadzama \& Happ, 1974) so that bursal mixing is likely to be similar in degree. The spermatheca in $T$. molitor is different in design to that of T. castaneum; there are many more storage tubules in T. molitor and these are much more elongate (Gage, 1992) so perhaps sperm storage patterns differ. It is not clear why P2 declines almost linearly to below 0.50 in $T$. molitor over 16 days since the competing matings (Siva-Jothy et al., 1996), yet there is no difference in the patterns when intermating intervals are either $10 \mathrm{~min}$ or $24 \mathrm{~h}$.

We do not find that third-male precedence values are significantly different from second-male (in the two-male experiments) values produced under the one-day inter-mating interval experiment and 
accordingly our results are consistent with the findings of Lewis \& Jutkiewicz (1998). Similar P3 and P2 results were found in the bruchid Callosobruchus maculatus (Eady \& Tubman, 1996). Our finalmale precedence results in the three-male experiments show characteristic high last-male precedence which begins to decline with time (Figure 3). Also, the first- and second-male values (in the triplemating experiments) show a weak increase over time. The results are consistent with interpretations of Bloch-Qazi et al. (1996) and Lewis \& Jutkiewicz (1998) that a small proportion of the inseminated ejaculate is accommodated in the limited-volume spermatheca and that last male's sperm gain primary precedence while earlier males' sperm become prevalent either via mixing or depletion of last-males' sperm. At present we are not able to say for either three-male or two-male sperm competition results whether previous males' sperm develop precedence with time beyond 30 days. If previous males' sperm develop precedence then the mechanism of sperm competition does not involve previous sperm passive or active loss from the system. Our overall results demonstrate a clear last male precedence that shows a variable decline with time, and which is influenced strongly by the inter-mating interval.

\section{Acknowledgements}

We thank Prof A. Sokoloff for unpublished information, F. Fleurat-Lessard and P. Golob for providing beetles, S. Wouters from Edialux for providing the technical malathion, Mrs J. Moreman for comments on the manuscript, and L. Van Belle and Y. Brostaux for statistical advice. This work was funded by the FRIA and the Royal Society; collaboration was made possible by the British Council, the FNRS and the CGRI.

\section{References}

Beeman, R.W., 1983. Inheritance and linkage of malathion resistance in the red flour beetle. Journal of Economic Entomology 76: 737-740.

Birkhead, T.R. \& A.P. Møller, 1998. Sperm Competition and Sexual Selection. Academic Press, London.

Bloch-Qazi, M., J.T. Herbeck, \& S.M. Lewis, 1996. Mechanism of sperm transfer and storage in the red flour beetle (Coleoptera: Tenebrionidae). Annals of the Entomological Society of America 89: 892-897.

Boorman, E. \& G.A. Parker, 1976. Sperm (ejaculate) competition in Drosophila melanogaster, and the reproductive value of females to males in relation to female age and mating status. Ecological Entomology 1: 145-155.

Cook, P.A., I.F. Harvey \& G.A. Parker, 1997. Predicting variation in sperm precedence. Philosophical Transactions of the Royal Society London B352: 771-780.

Dagnelie, P., 1998. Statistique Théorique et Appliquée. Tome 2 - Inférence Statistique à Une et à Deux Dimensions. De Boeck et Larcier, Paris.

Eady, P.E., 1994. Intraspecific variation in sperm precedence in the bruchid beetle Callosobruchus maculatus. Ecological Entomology 19: 11-16.

Eady, P.E. \& S. Tubman, 1996. Last-male sperm precedence does not break down when females mate with three males. Ecological Entomology 21: 303-304.

Eberhard, W.G., 1996. Female Control: Sexual Selection by Cryptic Female Choice. Princeton University Press.

Etman, A.A.M. \& G.H.S. Hooper, 1979. Sperm precedence of the last mating in Spodoptera litura. Annals of the Entomological Society of America 72: 119-120.

Gadzama, N.M. \& G.M.H. Happ, 1974. Structure and evacuation of the spermatophore of Tenebrio molitor.L. (Coleoptera: Tenebrionidae). Tissue and Cell 6: 95-108. 
Published in : Entomologia experimentalis et applicata (2001), vol. 99, iss. 1, pp. 55-64

Status : Postprint (Author's version)

Gage, M.J.G., 1992. Removal of rival sperm during copulation in a beetle, Tenebrio molitor. Animal Behaviour 44: 587-589.

Gwynne, D.T. \& A.W. Snedden, 1995. Paternity and female remat-ing in Requena verticalis (Orthoptera: Tettigoniidae). Ecological Entomology 20: 191-194.

Haubruge, E., L. Arnaud \& J. Mignon, 1997. The impact of sperm precedence in malathion resistance transmission in populations of the red flour beetle Tribolium castaneum (Herbst) (Col., Tenebrionidae). Journal of Stored Products Research 33: 143-146.

Haubruge, E., L. Arnaud, J. Mignon \& M.J.G. Gage, 1999. Fertilization by proxy: rival sperm removal and translocation in a beetle. Proceedings of the Royal Society London B 266: 1183-1187.

Lewis, S.M. \& S.N. Austad, 1990. Sources of intraspecific variation in sperm precedence in red flour beetles. American Naturalist 135: 351-359.

Lewis, S.M. \& S.N. Austad, 1994. Sexual selection in flour beetles: the relationship between sperm precedence and male olfactory attractiveness. Behavioral Ecology 5: 219-224.

Lewis, S.M. \& E. Jutkieweicz, 1998. Sperm precedence and sperm storage in multiply mated red flour beetles. Behavioral Ecology and Sociobiology 43: 365-369.

McKenzie, J.A. \& M. O’Farrell, 1993. Modification of developmental stability and fitness - malathionresistance in the Australian sheep blowfly, Lucilia cuprina. Genetica 89: 67-76.

Otronen, M., 1997. Sperm numbers, their storage and usage in the fly Dryomyza anilis. Proceedings of the Royal Society London B 264: 777-782.

Schlager, G., 1960. Sperm precedence in the fertilization of eggs in Tribolium castaneum. Annals of the Entomological Society of America 53: 557-560.

Simmons, L.W., 1987. Sperm competition as a mechanism of female choice in the field cricket, Gryllus bimaculatus. Behavioral Ecology and Sociobiology 21: 197-202.

Simmons, L.W. \& G.A. Parker, 1992. Individual variation in sperm competition success of the yellow dung fly, Scatophaga stercoraria. Evolution 46: 366-375.

Simmons, L.W. \& M.T. Siva-Jothy, 1998. Sperm competition in insects: mechanisms and the potential for selection. In: T.R. Birkhead \& A.P. Møller (eds), Sperm Competition and Sexual Selection. Academic Press, London, pp. 341-434.

Sinha, R.N., 1953. The spermatheca in the red flour beetle (Tri-bolium castaneum Herbst). Journal of the New York Entomological Society 41: 131-134.

Siva-Jothy, M.T. \& Y. Tsubaki, 1989. Variation in copula duration in Mnais pruinosa pruinosa Selys (Odonata: Calopterygidae). Behavioral Ecology and Sociobiology 24: 39-45.

Siva-Jothy, M.T. \& Y. Tsubaki, 1994. Sperm competition and sperm precedence in the dragonfly Nanophya pygmaea. Physiological Entomology 19: 363-366.

Siva-Jothy, M.T., D.E. Blake, J. Thompson \& J.J. Ryder, 1996. Short- and long-term sperm precedence in the beetle Tenebrio molitor: a test of the 'adaptive sperm removal' hypothesis. Physiological Entomology 21: 313-316.

Sokoloff, A., 1974. The Biology of Tribolium, Vol. II. Oxford University Press, Oxford.

Suzuki, N., T. Okuda \& H. Shinbo, 1996. Sperm precedence and sperm movement under different copulation intervals in the silkworm, Bombyx mori. Journal of Insect Physiology 42: 199-204.

Tschinkel, W.R., 1987. Fire ant queen longevity and age: estimation by sperm depletion. Annals of the 
Published in : Entomologia experimentalis et applicata (2001), vol. 99, iss. 1, pp. 55-64

Status : Postprint (Author's version)

Entomological Society of America 80: 263-266.

Ueno, H. \& Y. Ito, 1992. Sperm precedence in Eysacoris lewisi (Distant) (Heteroptera: Pentatomidae) in relation to duration between oviposition and the last copulation. Applied Entomological Zoology 27: $421-426$.

Waage, J.K., 1979. Dual function of the damsefly penis: sperm removal and transfer. Science 203: 916-918.

Ward, P.I., 1993. Females influence sperm storage and use in the yellow dung fly Scathophaga stercoraria (L.). Behavioral Ecology and Sociobiology 32: 313-319.

Wilson, N., S.C. Tubman, P.E. Eady \& G.W. Robertson, 1997. Female genotype affects male success in sperm competition. Proceedings of the Royal Society London B 264: 1491-1495.

Wool, D. \& O. Bergerson, 1979. Sperm precedence in repeated mating of adults of Tribolium castaneum (Coleoptera, Tenebri-onidae). Entomology Experimentalis et Applicata 26: 167-160.

Yamagishi, M., Y. Itô \& Y. Tsubaki, 1992. Sperm competition in the melon fly, Bratocera cucurbitae (Diptera: Tephritidae): effects of sperm longevity on sperm precedence. Journal of Insect Behaviour 5: 599-608.

Zeh, J.A. \& D.W. Zeh, 1994. Last-male sperm precedence breaks down when females mate with three males. Proceedings of the Royal Society London B 257: 287-292. 